DIVISION OF THE HUMANITIES AND SOCIAL SCIENCES CALIFORNIA INSTITUTE OF TECHNOLOGY

PASADENA, CALIFORNIA 91125

POLICY UNCERTAINTY, ELECTORAL SECURITIES AND REDISTRIBUTION

Andrea Mattozzi

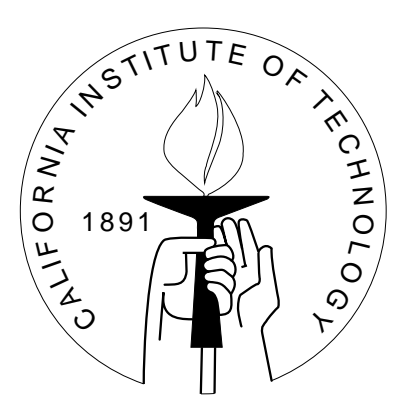

SOCIAL SCIENCE WORKING PAPER 1229

July 2005 


\title{
Policy Uncertainty, Electoral Securities and Redistribution
}

\author{
Andrea Mattozzi
}

\begin{abstract}
This paper investigates how uncertainty about the adoption of a redistribution policy affects political support for redistribution when individuals can trade policy contingent securities in the stock market. We show that the demand for redistribution is always smaller than in the case where no "policy-insurance market" is available. Consistent with the empirical evidence, our analysis implies that in economies with well-developed financial markets the level of redistribution decreases with the level of participation in these markets and with income inequality. We show that the existence of a policy insurance market may increase future expected inequality even if a majority of individuals are redistributing resources through private transfers.
\end{abstract}

JEL classification numbers: D72, G10, D31.

Key words: Policy Uncertainty, Financial Markets, Redistribution 


\title{
Policy Uncertainty, Electoral Securities and Redistribution *
}

\author{
Andrea Mattozzi
}

\section{Introduction}

The relationship between income distribution and citizens' demand for redistribution has long been a central issue in political economy. The seminal paper of Meltzer and Richard [16] presents a simple but powerful argument: the more positive-skewed the distribution of income, the higher the political support for redistributive taxation. However, this argument is not supported by the data. As pointed out by Bénabou [4] among others, for advanced countries the relationship runs in the opposite direction: societies that are more equal before redistribution tend to redistribute more rather than less.

In order to reconcile the basic insights of political-economy theories of redistribution with the empirical evidence, the recent literature has emphasized the potentially important role of individual uncertainty in explaining citizens' demand for redistribution. Bénabou and Ok [5], for example, show that if agents are uncertain about their prospect of future income and policies are persistent, relatively poor agents may choose to oppose a redistributive policy (see also Piketty [21]).

*This paper is a chapter of my doctoral dissertation. I am grateful to Bob Inman, Andrew Postlewaite, Frank Schorfheide, and in particular to Antonio Merlo for their comments and encouragement. I also benefitted from discussions with Mike Alvarez, Marco Cozzi, Federico Echenique, Jan Eeckhout, Jacob Goeree, Daniela Iorio, Matt Jackson, Dirk Krueger, Elena Pastorino and Nicola Persico. All usual disclaimers apply. 
A largely unexplored issue, however, is whether citizens' reaction to policy uncertainty plays any role in understanding the relationship between income distribution and the likelihood of adopting redistributive policies. Political uncertainty is a pervasive phenomenon which is inherent to the political process. It naturally arises because different candidates running for office, if elected, will implement different policies, and election results are uncertain; that is what happened, for example, in the last two Presidential elections in the United States. ${ }^{1}$ Furthermore, even after a candidate is elected, there might still be uncertainty about the likelihood that he will be able to implement his electoral promises (this is the case, for example, with the health care reform that was at the center of Clinton's 1992 Presidential campaign but was defeated in Congress during his presidency).

In this paper, I investigate how uncertainty about the adoption of a redistribution policy affects political support for redistribution when individuals can trade policycontingent securities. If individuals are risk averse and financial markets can provide insurance against policy uncertainty, redistribution will take place via the stock market, irrespective of whether the policy is implemented or not. Therefore, the existence of a "policy insurance market" will affect the equilibrium demand for redistribution policies, and, in turn, the likelihood that such policies will ultimately be implemented.

I propose an equilibrium model where agents who are ex-ante heterogeneous with respect to their income can trade policy-contingent securities and then vote on a redistribution policy whose probability of adoption increases with the number of its supporters. In this model, the very poor are excluded from the policy insurance market. The extent to which participation is constrained determines the "size" of the market. I show that as long as some individuals do not have access to the policy insurance market, the demand for redistribution is always smaller than in the case where no insurance is available. In equilibrium, relatively poor individuals receive private transfers from the rich through the insurance market, and oppose a public redistribution policy that they would otherwise have supported.

This model implies a relationship between income inequality and support for redis-

\footnotetext{
${ }^{1}$ For an extensive analysis of voters' information in U.S. Presidential elections see Alvarez [1].
} 
tribution. This relationship is non-monotonic and depends on the size of the policy insurance market. In particular, in economies with well-developed financial markets the level of redistribution decreases with the level of participation in these markets and with income inequality. Moreover, the size of the market also affects the inequality of the expected income distribution. In particular, I show that the existence of a policy insurance market may increase future expected inequality even if a majority of individuals are actually redistributing resources through private transfers. I use these theoretical findings to interpret the empirical evidence on the relationships between income inequality and redistribution.

The remainder of the paper is organized as follows. Section 2 discusses the related literature. Section 3 presents a simplified version of the model showing the main effect of the policy insurance market on individuals' behavior. Section 4 contains the general equilibrium model. Section 5 deals with the effects of the policy insurance market on the inequality of the expected income distribution after policy uncertainty is resolved. Section 6 explores the implications of the existence of a policy insurance market on the relationship between income inequality and popular support for redistribution. Section 7 concludes.

\section{$2 \quad$ Related Literature}

There are three strands of literature that are related to this paper. The first strand focuses on the interaction between politics and financial markets. Musto and Yilmaz [17] consider a model where two candidates with different ideologies compete for election by announcing redistribution policies and, unlike my model, all voters can share the "wealth risk" associated with different electoral outcomes by trading election-contingent securities in a frictionless financial market. They show that, since complete financial markets induce full insurance, all voters, regardless of their wealth, are indifferent between alternative redistribution policies. Hence, wealth considerations have no effect on electoral outcomes. Celentani et al. [7] analyze risk sharing and endogenous fiscal spending in the presence of complete markets. They show that if markets are sequentially complete, fiscal policy can be used to manipulate future security prices leading to inefficient equilibrium allocations. 
The second strand of literature presents different theoretical explanations for the fact that income inequality and popular support for redistribution are not positively correlated. Bénabou [4] shows that if redistribution is ex-ante welfare improving the support for redistribution is U-shaped in inequality. Lee and Roemer [13] show that public spending is not necessarily increasing in inequality since, as inequality rises, a given tax rate produces a smaller tax base.

The third strand of literature is on the empirical relationship between politics and the stock market. Mattozzi [14] shows that existing stocks that are currently traded on the U.S. stock market can be used to insure against political uncertainty. Focusing on the 2000 U.S. Presidential election, he constructs two "presidential portfolios" composed of selected stocks anticipated to fare differently under a Bush versus a Gore presidency. To construct these portfolios he uses data on campaign contributions by publicly traded corporations and identify the major contributors on each side (excluding corporations that made significant contributions to both candidates' campaigns). Using daily observations for the six months before the election took place, he shows that the excess returns of these portfolios with respect to overall market movements are significantly related to changes in electoral polls. Based on the evidence, he concludes that the presidential portfolios can actually be used as an instrument to hedge political uncertainty. Knight [12] tests whether policy platforms are capitalized into equity prices, using data from the 2000 U.S. Presidential election. He selects a sample of firms favored under the alternative policy platforms using reports from financial analysts, and shows that campaign platforms matter for firms' profitability. In a similar vein, Herron et al. [11] study the effect of the 1992 U.S. Presidential election outcome on the profitability of different economic sectors, and Ayers et al. [3] study whether security prices reflect fiscal policy uncertainty, using data from the same election. ${ }^{2}$

\footnotetext{
${ }^{2}$ At a more aggregate level, see also Pantzalis et al. [18], and Santa-Clara and Valkanov [22].

In light of the third strand of literature, it is worth noticing that, although betting on Presidential Elections is illegal in the U.S., in 2004 the Irish company Tradesports started offering a winner-take-all contract on the U.S. Presidential election.
} 


\section{An Example}

In this section I present a simple example in order to show two important results of this paper: First, as long as some individuals do not have access to the policy insurance market, the demand for redistribution is always smaller than in the case where no insurance is available. Second, an increase in income inequality may actually decrease the popular support for redistribution.

Consider a one-period model with three agents and logarithmic utility function over wealth. Each agent has an initial endowment of $y^{i}, i=\{1,2,3\}$, where $y^{1}=h, y^{2}=m$, and $y^{3}=l$. Assume that $h>m>l$, and $m<\frac{h+m+l}{3}=\bar{y}$ (that is, the median is below the mean). Agents have to choose between two different alternatives: the status quo, and a reform that taxes wealth proportionally at rate $\tau$ and redistributes $\tau \bar{y}$ to every agent. Sincere voting is weakly dominant with two alternatives, and since voting is costless, each agent will vote for the alternative that gives him higher utility. If indifferent between alternatives, agents randomize with equal probability.

Policy uncertainty is modeled by assuming that there is ex-ante uncertainty about the probability that the reform will be implemented. In particular, suppose that the probability of implementing the redistribution policy is equal to the share of its supporters in the population. ${ }^{3}$ Given that $m<\bar{y}$, the only agent that is strictly worse off with the reform is $y^{i}=h$. Therefore, the expected probability $q$ that the redistributive reform will be adopted is equal to $\frac{2}{3}$.

Suppose now that before voting over the reform a financial market is open. Agents can trade any quantity $b$ of a financial security that pays 1 if the reform is implemented and pays 0 otherwise. ${ }^{4}$ Let $p$ be the price of the bond.

The maximization problem that agents solve is:

$$
\max _{b_{y}} q \ln \left(\left(y^{i}+b_{y^{i}}(1-p)\right)(1-\tau)+\tau \bar{y}\right)+(1-q) \ln \left(y^{i}-b_{y^{i}} p\right),
$$

and market clearing requires:

$$
b_{l}+b_{m}+b_{h}=0
$$

\footnotetext{
${ }^{3}$ See the next Section for a discussion of this assumption.

${ }^{4}$ The argument is similar if instead $b$ pays 0 when the reform is implemented and 1 otherwise.
} 
where $b_{y^{i}}$ denotes the quantity of the security traded by the agent with initial endowment $y^{i}=\{l, m, h\}$. By taking first order conditions and using the market clearing condition I get ${ }^{5}$ :

$$
\begin{gathered}
b_{y^{i}}^{*}=\left(y^{i}-\bar{y}\right) \psi(\tau, q) \\
p^{*}=\frac{q(1-\tau)}{1-\tau q},
\end{gathered}
$$

where $\psi(\tau, q)$ is positive. ${ }^{6}$ Note that, since the security is a fair insurance and every agent can access the financial market, in equilibrium the wealth in the two states has to be the same. Every agent will be indifferent and the final probability of adopting the reform will depend on the tie-breaking rule. Moreover, irrespective of whether or not the reform is implemented, the ex post wealth distribution will be:

$$
y^{i}(1-\tau q)+\bar{y} \tau q, y^{i}=\{h, m, l\} .
$$

Therefore redistribution (through private transfers) will take place anyway. This result is similar to the one obtained by Musto and Yilmaz [17].

Consider now the case in which access to the financial market is conditional on having a given positive amount $\gamma$ of initial endowment. For example assume that the poorest agent $y^{i}=l$ cannot participate in the financial market $(l<\gamma<m)$. In this case, the new equilibrium will be:

$$
\begin{aligned}
b_{y^{i}}^{*} & =\left(y^{i}-\frac{m+h}{2}\right) \bar{\psi}(\tau, q, m, h, \bar{y}) \\
p^{*} & =\frac{q(1-\tau)}{1-\tau+\frac{2 \tau \bar{y}(1-q)}{m+h}}>\frac{q(1-\tau)}{1-\tau q}
\end{aligned}
$$

where $\bar{\psi}(\tau, q, m, h, \bar{y})$ is positive, and, in equilibrium, every agent that can access the market, namely $y^{i}=\{m, h\}$, will strictly prefer the status quo to the reform for any $\mathrm{q} ?(0,1) \cdot{ }^{7}$ Therefore, in equilibrium, the expected probability $\tilde{q}$ that the redistributive

${ }^{5}$ I assume $l>\frac{(m+h)(1-q) \tau}{3(1-q \tau)-(1-q) \tau}$ in order to rule out corner solutions.

${ }^{6}$ In this particular example,

$$
\psi(\tau, q)=\frac{\tau(1-\tau q)}{1-\tau}
$$

${ }^{7}$ In this particular example,

$$
\bar{\psi}(\tau, q, m, h, \bar{y})=\frac{2}{m+h} \frac{\left(1-\tau+\frac{2 \tau \bar{y}}{m+h}(1-q)\right) \tau \bar{y}}{\left(1-\tau+\frac{2 \tau \bar{y}}{m+h}\right)(1-\tau)}
$$


reform will be adopted is equal to $\frac{1}{3}$, that is strictly smaller than in the case where no insurance is available.

To see the intuition behind this result note that if everybody has access to the financial market, the $h$ agent can buy out $m$ and $l$, by making them at most indifferent between the two policies. If the poor agent cannot access the market, the resulting decline in supply will increase the equilibrium price. At the new price agent $m$ is more than compensated by private transfers, and therefore she strictly prefers the status quo scenario. In other words, when access to market is unrestricted, all the risk is idiosyncratic and therefore insurable. Whereas if agent $l$ cannot insure herself part of the risk becomes systematic. In the redistribution state, the $l$ agent is extracting money from the two other agents, and they will therefore strictly prefer the status quo.

Suppose now, that holding the mean $\bar{y}$ constant, $h$ decreases to $h^{\prime}<h$ and $l$ increases to $l^{\prime}>\gamma>l$. As a result, the popular support for redistribution and therefore the probability of adopting the redistribution will increase even if the initial distribution of endowments is more equal. ${ }^{8}$

In the following three sections I show that the conclusions obtained above hold in a more general environment and explore the relationship between inequality and the adoption of redistributive policies.

\section{The Model}

There is a continuum of agents of measure one. Each agent is endowed with pre-tax income $y \geq 0$ that is an independent draw from a known distribution $F$ with density $f$ and support $Y$. Let

$$
\bar{y}=\int_{Y} y d F(y)
$$

be the mean income.

Agents are called to vote on the adoption of a reform. Two alternatives are available:

\footnotetext{
${ }^{8}$ Clearly, in this simple example, the result depends on the tie-breaking rule.
} 
the status quo versus a policy $(\tau, T)$, where $\tau$ is a proportional tax on wealth and $T$ is a per-capita lump sum transfer. I assume that the budget is balanced, that is $T=\tau \bar{y}$.

Following most of the literature on redistributive taxation, I use a simple linear tax as a convenient way to describe my results. The implications of our analysis are, however, more general and hold for a larger class of policies entailing a conflict of interest between those who gain from it and those who are hurt by it.

As in the previous section, policy uncertainty is modeled by assuming that there is ex-ante uncertainty about the probability that the reform will be implemented. Namely, I assume that the probability of implementing the redistribution policy increases monotonically with the number of its supporters. In other words, for given proportion $q$ of individuals in favor of the redistributive policy, the probability that this policy will be actually implemented is $\varphi(q) \in[0,1]$, where $\varphi\left(\frac{1}{2}\right)=\frac{1}{2}$, and $\frac{\partial \varphi(q)}{\partial q}>0 .{ }^{9}$ As long as $\varphi(q)$ is continuous and strictly increasing, its particular shape is not going to affect qualitatively the results. Therefore, for simplicity, let $\varphi(q)$ be the identity function. ${ }^{10}$ The ex-ante probability of adopting the redistribution policy is:

$$
q=F(\bar{y})
$$

Before elections are held, a financial market opens. Agents can trade any quantity $b$ of a security that pays 1 when the reform is enacted and 0 otherwise. Let $p$ be the security price and $\gamma>0$ be an exogenous threshold on pre-tax income above which agents have access to the market. ${ }^{11}$ This assumption has the merit of making transparent the force that is driving the result, and will be relaxed later by considering the existence of an expost individual budget constraint on the amount of securities that can be traded. Finally, I assume that the utility function $U(x)$ belongs to the class of hyperbolic absolute risk

\footnotetext{
${ }^{9} \mathrm{I}$ borrow this modelling assumption from Grossman and Helpman [8].

${ }^{10} \mathrm{An}$ alternative way to model policy uncertainty is to borrow from the probabilistic voting literature, and assume the existence of an individual-specific ideological bias (in favor or against redistribution) that is drawn from a known distribution.

${ }^{11}$ Guiso et al. [9][10] show that the proportion of US households investing in risky assets, with gross financial wealth falling in the lowest quartile, is $1.4 \%$. This proportion is less than $1 \%$ for the UK, the Netherlands, and Italy, and less than $3 \%$ for Germany. If we consider direct and indirect stockholding, all figures are below $5 \%$, with the exception of Germany at $6.6 \%$.
} 
aversion (HARA), and it is strictly concave. In particular, I assume that wealth level $x$, gives the agent a utility of:

$$
\begin{gathered}
U(x)=\frac{1-\theta}{\theta}\left(\frac{\alpha x}{1-\theta}+\beta\right)^{\theta} \\
\frac{\alpha x}{1-\theta}+\beta>0
\end{gathered}
$$

and strict concavity requires:

$$
\alpha>0
$$

Constant absolute risk aversion, constant relative risk aversion, and logarithmic utility specifications are special cases of the HARA utility. ${ }^{12}$

The maximization problem that agents solve is ${ }^{13}$ :

$$
\max _{b} q U((y+b(1-p))(1-\tau)+\tau \bar{y})+(1-q) U(y-b p)
$$

Market clearing requires:

$$
\int_{Y \mid y>\gamma} b d F(y)=0 .
$$

$U(x)$ belonging to the class of HARA utility function is a sufficient condition for uniqueness of equilibrium. Therefore, in the unique equilibrium:

$$
\begin{gathered}
b^{*}(y)=(y-\tilde{y}) \frac{\tau}{1-\tau} \frac{\alpha \bar{y}+\beta(1-\theta)}{\alpha \tilde{y}+\beta(1-\theta)} \frac{(1-q) h^{1-\theta}+q(1-\tau)}{(1-q) h^{1-\theta}+q h} \\
p^{*}=\frac{q(1-\tau)}{(1-q) h^{1-\theta}+q(1-\tau)}
\end{gathered}
$$

where

$$
h=\frac{\alpha(\tilde{y}(1-\tau)+\tau \bar{y})+\beta(1-\theta)}{\alpha \tilde{y}+\beta(1-\theta)},
$$

\footnotetext{
${ }^{12}$ If $\beta=1$ and $\theta \rightarrow-\infty$, we have the CARA utility, if $\beta \rightarrow 0, \alpha=1-\theta$, the CRRA utility obtains. If $\beta \rightarrow 0, \theta \rightarrow 0$ we have the logarithmic utility specification.

${ }^{13}$ Notice I am not imposing any constraint on the maximization problem. This implies in principle that agents can sell an amount of securities greater than their pre-tax endowment and end up paying a negative tax. As long as $\gamma$ is not too small this will never happen in equilibrium (see the Appendix for the general case).
} 
and

$$
\begin{gathered}
\tilde{y}=\frac{\int_{Y \mid y>\gamma} y d F(y)}{\int_{Y \mid y>\gamma} d F(y)} \geq \bar{y}, \\
\tilde{y}>\gamma .
\end{gathered}
$$

Finally, note that

$$
\tilde{y}>\gamma
$$

I can use the equilibrium characterization to prove the following proposition:

Proposition 1. For any continuous distribution $F(y)$, all active agents on the market $(y \geq \gamma)$ strictly prefer the status quo.

Proof. All agents participating in the market strictly prefer the status quo if and only if:

$$
U\left(y-b^{*}(y) p\right)>U\left(\left(y+b^{*}(y)(1-p)\right)(1-\tau)+\tau \bar{y}\right) .
$$

By using (1), I can rewrite the above inequality as

$$
p>\frac{q(1-\tau)}{1-q \tau} .
$$

By using (2), in the unique equilibrium it will be

$$
p^{*}>\frac{q(1-\tau)}{1-q \tau},
$$

if and only if

$$
h<1 \text {. }
$$

But since

$$
\tilde{y} \geq \bar{y}
$$

$h$ is always smaller than 1.

In order to capture the intuition behind this result, consider Figures 1 and 2. Figure 1 depicts the demand schedule $b^{*}(y)$ as a function of $y$, in the case of $U(x)=\frac{x^{1-\alpha}-1}{1-\alpha}, \alpha=2$, $\tau=\frac{1}{2}, y \sim U[0,1]$ and $\gamma=\left\{0, \frac{1}{4}\right\}$. It shows how agents react to policy uncertainty: rich (poor) agents buy (sell) a positive amount of securities. Since $\tilde{y}$ is the mean of the income distribution truncated at $\gamma$ and, in equilibrium, it is equal to the income level of 
the indifferent agent on the market, it follows that $b^{*}(\tilde{y})=0$. Note that $\tilde{y}$ is increasing in $\gamma$, and an increase in $\gamma$ induces a reduction, in equilibrium, of the total amount of securities traded and therefore a reduction of the size of the market. If individuals with incomes in the left tail of the distribution cannot access the market, the resulting excess in demand will increase the security price. This is the reason why the two schedules depicted in Figure 1 do not overlap. The result stated in Proposition 1 follows from the fact that, at the new equilibrium price, all agents trading in the market whose income is below the mean income, are more than compensated by private transfers, and therefore they strictly prefer the status quo scenario.

Figure 2 depicts the difference $D U$ in utility between the status quo and the redistribution state as a function of $y$, and $\gamma$, using the same parametrization of Figure 1 . When $\gamma=1$ (no trading at all), $D U$ is monotonically increasing, and $D U(\bar{y})=0$. When $\gamma=0$ (unrestricted trading), $D U$ is equal to zero for all $y$. The most interesting case is when $\gamma \in(0,1)$. For low values of $y, D U$ is negative and increasing. For values of $y$ greater than $\gamma$ (set equal to $\frac{1}{4}$ in the picture), $D U$ is always positive. The chosen parametrization affects the shape of $D U$ but not its quasi-monotonicity property. ${ }^{14}$ Given that every agent that can trade in the market will strictly prefer the status quo policy, the resulting expected probability $\tilde{q}$ of implementing the redistributive policy will be:

$$
\tilde{q}=\min \{F(\gamma), F(\bar{y})\} \leq q
$$

Therefore, Proposition 2 follows:

Proposition 2. For any continuous distribution $F(y)$, the ex-post probability $\tilde{q}$ of adopting redistribution is (weakly) smaller than the ex-ante probability $q$, and an increase in the size of market (a decrease in $\gamma$ ) decreases the support for the reform.

Figure 2 offers an alternative way to capture the economic intuition behind this result. Consider the problem faced by the marginal agent that can trade in the market in the

\footnotetext{
${ }^{14}$ For example in the case of

$$
U(x)=\ln x,
$$
}

$D U$ is flat for $y>\gamma$. 
most interesting case of $\gamma<\bar{y}$, that is the marginal agent is relatively poor and hence, in equilibrium, he will sell a positive amount of securities. If the redistributive policy is not implemented, he will receive a private transfer from rich agents that is compensating him for not having his ex-ante most preferred alternative. If the redistributive policy will be the selected alternative, he has to make a private transfer to rich individuals. However, this transfer has to compensate the rich agents also for the additional amount of redistribution that is due to the fact that the very poor agents cannot trade in the market. Therefore, in equilibrium, he will strictly prefer to vote for his ex-ante least preferred alternative.

As I already pointed out, assuming an exogenous $\gamma$ has the merit of making transparent the force that is driving the result. However, the result of Proposition 2 continues to hold even if I drop the assumption of an exogenous threshold on pre-tax income above which agents have access to the market. In particular, in the Appendix, I explicitly consider the existence of an ex-post individual budget constraint on the amount of securities that can be traded, and set $\gamma=\underline{y}$, where $\underline{y}$ is the lower bound of $Y$. (i.e. unrestricted access to the financial market).

Finally, note that under the assumption of an exogenous $\gamma$, the equilibrium value of $\tilde{q}$ does not depend on $q$. This is not generally true when we relax this assumption. Nonetheless, it can be shown that, also in the more general case, there exists a unique equilibrium in which the proportion of agents in favor of redistribution is always strictly smaller than in the case where agents do not react to electoral uncertainty (see Appendix).

The main results of this section are that as long as poor individuals are constrained in policy insurance market, the demand for redistribution is always smaller than in the case where no insurance is available, and the size of the market is positively correlated with the probability that a redistributive reform is adopted. In the next section I show that the size of the market also affects the inequality of the expected income distribution, and the existence of a policy insurance market may increase future expected inequality even if a majority of individuals are actually redistributing resources through private transfers. 


\section{Expected Income Distribution}

In this model, private redistribution takes place before the election even in the case where the reform is ultimately not implemented. The objective of this section is to analyze the effect of the policy insurance market on the inequality of the expected income distribution after policy uncertainty is resolved.

I can compare the degree of inequality of different income distributions using the concept of second order stochastic dominance. $F_{x}$ is more unequal than $F_{y}$, if $F_{x}$ is a mean preserving spread of $F_{y}$. More formally, let $X$ be the common support of $F_{y}$ and $F_{x}$, then $F_{x}$ is more unequal than $F_{y}$ if:

$$
\int_{X} x d F_{x}=\int_{X} x d F_{y}
$$

and

$$
\int_{\underline{x}}^{x}\left(F_{x}-F_{y}\right) d s \geq 0 \text { for all } x \in X,
$$

where $\underline{x}$ is the lower bound of $X$. The equality of means implies that:

$$
\int_{X} F_{x}=\int_{X} F_{y}
$$

Hence if $F_{x}$ and $F_{y}$ cross only once, (1) is satisfied.

Let $z$ be the expected income after elections without market:

$$
z=q \tau \bar{y}+(1-q \tau) y
$$

By using the convolution formula:

$$
F_{z}=\left\{\begin{array}{cc}
F\left(\frac{z-q \tau \bar{y}}{1-q \tau}\right) & \text { for } z \geq q \tau \bar{y}+(1-q \tau) \underline{y} \\
0 & \text { otherwise }
\end{array}\right.
$$

where $\underline{y}$ is the lower bound of $Y$. Since $F$ and $F_{z}$ have the same mean, $F(\underline{y})>F_{z}(\underline{y})=0$, and they cross only once, $F$ is a mean preserving spread of $F_{z}$. I will use $F_{z}$ as a benchmark to evaluate the effect of the market in terms of expected income distribution.

I have to consider two cases: 


\section{Case $1 \gamma \geq \bar{y}$}

In this case the electoral market redistributes income through private transfers but does not affect the probability of adopting the reform. Let $z_{m}$ be the expected income after elections with market, and $F_{z_{m}}$ be the expected income distribution.

Proposition 3. If $\gamma \geq \bar{y}$ the introduction of the electoral market decreases expected income inequality for any continuous initial distribution $F$.

The proof can be found in the Appendix. Figure 3 provides the intuition. $F_{z_{m}}$ and $F_{z}$ have the same support and are identical for $y<\gamma$. Since they have the same mean, the areas denoted by $A$, and $B$ in Figure 3 are equivalent, therefore $F_{z}$ is a mean preserving spread of $F_{z_{m}}$.

Case $2 \gamma \in(\underline{y}, \bar{y})$

In this case the effect of the electoral market is twofold: it redistributes income through private transfers before the reform takes place, and it affects the probability of adopting the reform. The next proposition provides a sufficient condition under which the latter effect is dominant and the conclusion of Proposition 3 is reversed.

Proposition 4. There exists a $\gamma^{*} \in(\underline{y}, \bar{y})$, such that if $\gamma \leq \gamma^{*}$ the introduction of the electoral market increases expected income inequality for any continuous initial distribution F.

The proof is in the Appendix. Figure 4 provides a graphical intuition in the case of $\gamma \leq \gamma^{*}$. If $\gamma \leq \gamma^{*}$ it is possible to show that $F_{z_{m}}$ and $F_{z}$ cross only once. Since they have the same mean, it follows that $F_{z_{m}}$ is a mean preserving spread of $F_{z}$.

When the financial market is widely accessible, a large redistribution is actually taking place before the election, and this maps in a smaller support for the redistribution state. Therefore we should expect the policy insurance market to have a positive effect of on expected inequality. This section demonstrates, however, that this might not be the case. Even if a large proportion of agents is moving resources from one state to the other, as long as the lower tail of the distribution is still completely exposed to the electoral risk, the gap between losers and winners widens and, moreover, there is a shift of probability 
mass on the status quo. When the policy insurance market is large, agents' reaction to electoral uncertainty leads, in expectation, to a distribution even less equal.

\section{Income Inequality and Redistribution}

In this section, I study the relationship between income inequality and redistribution in an economy where the income distribution is positively-skewed. In particular, to analyze the effect of the initial income distribution on the expected probability $\tilde{q}$ of implementing the redistributive policy, assume that income is drawn from a Pareto distribution with parameters $c>0$ and $\Delta<1 .^{15}$ Under these assumptions we have that:

$$
\begin{aligned}
F(y) & =1-\left(\frac{c}{y}\right)^{\frac{1}{\Delta}}, \\
\bar{y} & =\frac{c}{1-\Delta} \\
\frac{\bar{y}}{y_{\text {med }}} & =\frac{1}{(1-\Delta) 2^{\Delta}}, \\
\text { Gini } & =\frac{\Delta}{2-\Delta} .
\end{aligned}
$$

In the case without a policy insurance market, the probability of adopting the reform is given by:

$$
q=F(\bar{y})=1-(1-\Delta)^{\frac{1}{\Delta}}
$$

An increase in $\Delta$, which increases the Gini index as well as the ratio between mean and median income, unambiguously increases $q$. This is what a median voter model would have predicted. A reform with asymmetric benefit will be more popular the more polarized a society is, provided that a majority of voters was already in favor of it.

The comparative statics of the model are quite different if I consider the presence of the market. Indeed, for $\gamma>c$, the end of period probability of implementing the reform

\footnotetext{
${ }^{15}$ The same analysis holds if I assume a log normal distribution. The log normal distribution is a reasonable approximation of the US empirical distribution of income. The Pareto distribution is a reasonable approximation of the empirical distribution of high incomes. See Lee and Roemer [13] and Bénabou [4].
} 
is now given by the following expression:

$$
\tilde{q}=\left\{\begin{array}{cc}
1-\left(\frac{c}{\gamma}\right)^{\frac{1}{\Delta}} & \gamma<\frac{c}{1-\Delta} \\
1-(1-\Delta)^{\frac{1}{\Delta}} & \gamma>\frac{c}{1-\Delta}
\end{array}\right.
$$

and, therefore, Proposition 5 follows:

Proposition 5. An increase in inequality will decrease (increase) the expected probability of adopting the reform if and only if $\gamma<(>) \bar{y}$. In particular:

$$
\frac{d \tilde{q}}{d \Delta}=\left\{\begin{array}{cc}
\frac{\left(\frac{c}{\gamma}\right)^{\frac{1}{\Delta}}}{\Delta^{2}} \ln \frac{c}{\gamma}<0 & \gamma<\bar{y}=\frac{c}{1-\Delta} \\
(1-\Delta)^{\frac{1-\Delta}{\Delta}} \frac{(1-\Delta) \ln (1-\Delta)+\Delta}{\Delta^{2}}>0 & \gamma>\bar{y} .
\end{array}\right.
$$

Increasing inequality has two distinct effects on the political support for redistribution: (i) given the distributional assumptions, it shifts probability mass towards below-average income, (ii) for given $\gamma$, it affects the fraction of agents who trade in the market. The first effect increases the value of $\tilde{q}$, the second effect decreases the value of $\tilde{q}$. In the case in which relatively poor individuals participate in the market, i.e. $\gamma<\bar{y}$, the second effect dominates, and an increase in inequality decreases the likelihood of adopting the reform. When $\gamma$ gets larger, the existence of insurance only affects rich individuals that were already against redistribution. Hence, the first effect is the dominant one, and an increase in inequality is positively correlated with the likelihood of adopting the redistributive reform. Furthermore, notice that, since in the case of a Pareto distribution $\bar{y}=\frac{c}{1-\Delta}$ is increasing in $\Delta$, for fixed $\gamma$ we can always find a $\widetilde{\Delta}$ such that $\gamma=\frac{c}{1-\widetilde{\Delta}}$. Therefore, for intermediate values of $\gamma$, the relationship between income inequality and support for redistribution may be non-monotonic.

Figure 5 depicts the relationship between income inequality as measured by $\Delta$, and the equilibrium probability $\tilde{q}$ of adopting the reform for different values of $\gamma$.

The relationship between income inequality and support for redistribution depends crucially on the size of the policy insurance market. If financial markets can provide insurance against policy uncertainty, Proposition 5 implies that in economies with welldeveloped financial markets the level of redistribution decreases with the level of participation in these markets and with income inequality. The latter result provides a possible 
explanation for the empirical observation that, among advanced economies, countries that are more equal before redistribution tend to redistribute more rather than less. This is illustrated in Figure 6, that depicts a cross-country scatter plot of the relationship between Gini index and the ratio to GDP of transfers to households for OECD economies. $^{16}$

Finally note that Proposition 5 implies that, for a given level of inequality in the income distribution, different countries can support very different level of redistribution depending on the development of their financial institutions. A failure of not taking into account this aspect may lead to a misspecified empirical model of the relationship between inequality and redistribution. This is due to the fact that we are mixing regimes. In particular, this may provide an explanation of why Perotti [19] among others finds no significant empirical relationship between inequality and the share of transfers or government expenditures in GDP, when he considers a sample of developed and developing countries.

\subsection{Discussion}

In the previous section I have shown that a non-monotonic relationship between inequality and popular support for redistribution is obtained in the case of an increase in the Gini index, or in the ratio between mean and median income. This particular increase in inequality is clearly not a mean preserving spread. In general, in any standard median voter type of model, a mean preserving spread in the distribution of income can hardly induce a negative relationship between inequality and redistribution. Furthermore, even if the redistribution is ex-ante welfare improving, a symmetric, mean preserving spread in any symmetric distribution of income cannot generate a non-monotonic relationship. ${ }^{17}$

\footnotetext{
${ }^{16}$ Data are taken from the OECD Economic Outlook and Deninger and Squire dataset (1996), available at URL: http://www.worldbank.org/research/growth/dddeisqu.htm

In light of the existing literature on the relationship between different electoral systems and the size of the redistributive sector (see, e.g. Austen-Smith [2]), it is worth noting that a similar picture also obtains if I restrict attention to parliamentary democracies with a proportional electoral system.

${ }^{17} \mathrm{~A}$ symmetric, mean preserving spread leads to a decline in popular support for an ex-ante welfare improving redistribution, and to an increase in popular support for a redistribution that entails some
} 
This is also true in the present model under the assumption of an exogenous threshold on pre-tax income above which agents have access to the market. However, in this subsection I show that in the more general and realistic case where agents have an ex-post individual budget constraint on the amount of securities that can be traded, and I set $\gamma=\underline{y}$ (i.e. unrestricted access to the financial market), I can easily construct examples where even a symmetric mean preserving spread in the distribution of income can generate a nonmonotonic relationship between inequality and support for redistribution.

Assume that income $y$ is distributed in $[0,1]$ with density function $f(y)$, where

$$
f(y)=\left\{\begin{array}{cc}
\frac{1}{2} & \text { if } y \in\left[0, \frac{1}{2}-\varepsilon\right] \\
\frac{1}{2}+\frac{1}{4 \varepsilon} & \text { if } y \in\left(\frac{1}{2}-\varepsilon, \frac{1}{2}+\varepsilon\right) \\
\frac{1}{2} & \text { if } y \in\left[\frac{1}{2}+\varepsilon, 1\right]
\end{array}\right.
$$

and $\varepsilon \in\left(0, \frac{1}{2}\right)$. Then

$$
\begin{gathered}
\bar{y}=\int_{0}^{1} y d F(y)=\frac{1}{2} \\
\operatorname{Var}(y)=\int_{0}^{1}(y-\bar{y})^{2} d F(y)=\frac{1+4 \varepsilon^{2}}{24} .
\end{gathered}
$$

Therefore, an increase in $\varepsilon$ represents a mean preserving spread in the distribution. For simplicity let $\tau=\frac{1}{2}$. Finally, assume a logarithmic utility function. The problem that agents solve is:

$$
\begin{gathered}
\max _{b} q \ln \left((y+b(1-p)) \frac{1}{2}+\frac{1}{4}\right)+(1-q) \ln (y-b p) \\
\text { s.t. } y+b(1-p) \geq 0
\end{gathered}
$$

and market clearing requires:

$$
\int_{0}^{1} b d F(y)=0 .
$$

In equilibrium

deadweight loss. See Bénabou [4], page 100, footnote 7 . 


$$
b^{*}=\left\{\begin{array}{cl}
\frac{2(q-p) y-p(1-q)}{2 p(1-p)} & y>\frac{p(1-q)}{2 q} \\
-\frac{y}{1-p} & y<\frac{p(1-q)}{2 q} .
\end{array}\right.
$$

In order to solve for the equilibrium price, I have to consider two cases: i) $\frac{p(1-q)}{2 q} \in$ $\left(\frac{1}{2}-\varepsilon, \frac{1-q}{2}\right)$, and ii) $\frac{p(1-q)}{2 q} \leq \frac{1}{2}-\varepsilon$.

In case i), the market clearing condition delivers

$$
p(q)=\frac{q\left(1-q+(7-3 q) 2 \varepsilon-\sqrt{2 \varepsilon} \sqrt{84 \varepsilon-6 q-52 q \varepsilon+7-(q+(1-q) 2 \varepsilon)^{2}}\right)}{(1+2 \varepsilon)(1-q)^{2}},
$$

and if $\frac{1-p(q)}{4} \leq \frac{1}{2}-\varepsilon$, it follows that $\tilde{q}$ is the unique solution to

$$
\tilde{q}-\frac{1-p(\tilde{q})}{8}=0
$$

It is possible to show numerically that there exist $\varepsilon^{\prime}$, and $\varepsilon^{\prime \prime}$ such that for $\varepsilon \in\left(\varepsilon^{\prime}, \varepsilon^{\prime \prime}\right)$, the support for redistribution is decreasing in the level of inequality, that is $\frac{\partial \tilde{q}}{\partial \varepsilon}<0$. The opposite relation obtains if $\varepsilon \in\left(\varepsilon^{\prime \prime}, \frac{1}{2}\right)$. In case ii), it is a matter of simple algebra to show that $\tilde{q}$ is independent of $\varepsilon$.

The intuition for this result is that, when $\varepsilon \in\left(\varepsilon^{\prime}, \varepsilon^{\prime \prime}\right)$, an increase in inequality increases the mass of constrained agents in the market, and the equilibrium price increases to compensate the resulting excess of demand. The agent that was indifferent between the redistributive policy and the status quo (that in equilibrium is always a constrained net seller of electoral securities as it is shown in the Appendix), is now strictly better off if the redistributive policy is not implemented. By further increasing inequality above $\varepsilon^{\prime \prime}$, the effect on prices is compensated by the increase in the mass of low income individuals and the relation between inequality and support for redistribution becomes positive. Finally, if $\varepsilon$ is very small, that is a large fraction of the population is concentrated around the mean, an increase in inequality has no effect on the mass of constrained sellers and, in this particular example, $\tilde{q}$ is independent of $\varepsilon$. 


\section{Conclusion}

This paper presents a model that demonstrates the role citizens' reactions to policy uncertainty plays in the demand for redistributive policies. I show how ex-ante uncertainty about a government policy with redistributive consequences will influence the proportion of agents in favor of it, if a subset of the agents can trade policy-contingent securities. In an economy with a policy insurance market, there is less demand for redistribution than in one without such a market, and demand for redistribution may decrease as income inequality increases. I provide conditions under which the existence of a policy insurance market increases future expected income inequality even if a large proportion of agents is redistributing resources through private transfers.

Based on the prediction of the theoretical model, my analysis implies that the level of participation in the stock market should be negatively related to support for policies with redistributive content. This result is consistent with several empirical observations. First, European countries are characterized by smaller participation in the stock market and larger redistributive transfers with respect to the U.S.. ${ }^{18}$ Furthermore, among all OECD economies, countries with a higher ratio to GDP of total stock market value traded, are also characterized by a smaller level of transfers to households as a share of the GDP, as shown in Figure $7 .^{19}$

By focusing on the recent U.S. experience, two important facts can be singled out. First, stock ownership in the U.S. has changed dramatically in the last decade. The proportion of U.S. households owning stocks directly or indirectly (through mutual funds or retirement accounts) has risen from 31.6 percent in 1989 to 51.9 percent in 2001, and the median income of stock owners has decreased by more than 9 percent in the period 1989-1998. ${ }^{20}$ Second, the U.S. level of transfers to households as a share of the GDP has almost steadily decreased in the nineties'. A similar picture emerges by considering broader aggregates like the share of total government expenditures over GDP. Moreover, taking for granted the conventional wisdom that democratic platforms tend to carry more

\footnotetext{
${ }^{18}$ See Guiso et al. [10].

${ }^{19}$ Data are taken from OECD Economic Outlook, and Beck, Demirgüç-Kunt, and Levine data set (2001).

${ }^{20}$ Data are taken from the Survey of Consumer Finances. See also Bertaut and Starr-McCluer [6].
} 
redistributive spending, the share of democratic votes in House elections has decreased from 52 percent in 1990 to 47 percent in 2000. Given that the nineties' were also characterized by an increasing inequality in income distribution, the combination of increasing stock market participation and decreasing support for redistribution is consistent with the mechanism described in the theoretical model. ${ }^{21}$

Clearly, any relationship between stock market participation and preference for redistribution that can be inferred from aggregate data may turn out to be spurious and due to a variety of other phenomena. However, the National Election Study (NES) data seems a promising starting point for further empirical analysis using micro level data. The latest waves of the NES contain both individual level information about stock market participation, and variables that summarize individual preferences on whether the government should increase or decrease the level of service/spending. ${ }^{22}$ It is interesting to note that in every income quintile the proportion of stock owners who prefer less than or equal government services and spending with respect to the status quo, is systematically higher than the proportion of those without stocks. In addition, even after controlling for sex, race, age, education, and income, a significative negative correlation between stock ownership and support for policies with redistributive content is observed. ${ }^{23}$ This is only preliminary evidence, but it suggests that an economy's financial structure appears to be correlated with its policy choices in a way that is consistent with our theoretical model.

\footnotetext{
${ }^{21}$ Data are taken from Economic Outlook. The increasing income inequality that characterized the US economy in the 90s has been documented by Krugman (New York Times, October 20, 2002).

${ }^{22}$ Alternatively, one could look at preferences revealed by the vote cast in the election. There are two problems in using this alternative approach. First, one should take into account the selection bias due to the choice of voting versus abstention in the election. Second, it is not clear which type of election one should focus on, or how incumbency effects should be treated.

${ }^{23}$ See also Mattozzi [14].
} 


\section{Appendix}

In the paper all results were derived in the case of exogenously restricted access to the market and without taking into account the possibility that agent's budget constraint was binding. Here I show that the results derived in the previous sections are robust to the introduction of a budget constraint, and I set $\gamma=\underline{y}$ (i.e. unrestricted access to the financial market). The maximization problem is:

$$
\begin{gathered}
\max _{b} \frac{1-\theta}{\theta}\left(q\left(\frac{\alpha((y+b(1-p))(1-\tau)+\tau \bar{y})}{1-\theta}+\beta\right)^{\theta}+(1-q)\left(\frac{\alpha(y-b p)}{1-\theta}+\beta\right)^{\theta}\right) \\
\text { s.t. } y+b(1-p) \geq 0, \\
y-b p \geq 0, \\
\text { and } \int_{Y} b d F(y)=0,
\end{gathered}
$$

where the two inequalities represent the budget constraint (for sellers and buyers respectively), and the last equation is the market clearing condition. I will solve the problem by first assuming that the second constraint $(y-b p \geq 0)$ does not bind, and then verify that this is always true in equilibrium. FOCs deliver

$$
b^{*}(y)=\left\{\begin{array}{cc}
\frac{(x-(1-\tau)) \alpha y-\alpha \bar{y} \tau-x \beta(1-\theta)(1-x)}{p x \alpha+\alpha(1-p)(1-\tau)} & y \geq s \\
-\frac{y}{1-p} & y<s
\end{array},\right.
$$

where

$$
\begin{gathered}
x=\left(\frac{q(1-p)(1-\tau)}{(1-q) p}\right)^{\frac{1}{1-\theta}}, \\
s=\frac{\alpha \bar{y} \tau+x \beta(1-\theta)(1-x)}{x \alpha}(1-p) .
\end{gathered}
$$

In equilibrium, the market clearing condition is

$$
\begin{gathered}
Q(p)=\int^{s}\left(-\frac{y}{1-p}\right) d F(y)+ \\
+\int_{s} \frac{(x-(1-\tau)) \alpha y-\alpha \bar{y} \tau-x \beta(1-\theta)(1-x)}{p x \alpha+\alpha(1-p)(1-\tau)} d F(y)=0 .
\end{gathered}
$$

Note that a necessary condition for an equilibrium to exist is that $x>(1-\tau)$ (otherwise $Q(p)<0)$. This implies that

$$
p<\bar{p}=\frac{q}{q+(1-q)(1-\tau)^{-\theta}}<q,
$$


and

$$
Q(\bar{p})<0 \text {. }
$$

Moreover, if $p$ goes to 0 ,

$$
\lim _{p \rightarrow 0} Q(p)>0
$$

Since $Q(p)$ is continuous in $p$, an equilibrium always exists. By evaluating $\frac{d(Q(p))}{d p}$ in $Q(p)=0$ :

$$
\left.\frac{d Q(p)}{d p}\right|_{Q(p)=0}=\frac{-x \int^{s} \frac{y}{(1-p)^{2}} d F(y)+\frac{d x}{d p} \int_{s} \phi(y, p) d F(y)}{p x+(1-p)(1-\tau)},
$$

where

$$
\phi(y, p)=\frac{(1-\tau) \alpha y+\beta(1-\theta)\left(p x^{2}+(1-p)(1-\tau)(2 x-1)\right)+\alpha \bar{y} \tau p}{p x \alpha+\alpha(1-p)(1-\tau)}
$$

is increasing in $y$, and

$$
\frac{d x}{d p}=-\frac{(1-\tau) q}{(1-\theta)(1-q) p^{2}} x^{\theta}<0
$$

Since

$$
\int_{s} \phi(y, p) d F(y)>\phi(s, p)(1-F(s))>0
$$

it follows that

$$
\left.\frac{d Q(p)}{d p}\right|_{Q(p)=0}<0
$$

and, therefore, the equilibrium price is unique. Note that, for $y \geq s, y-b^{*}(y) p$ is increasing in $y$, and

$$
s-b^{*}(s) p=s \frac{1}{1-p}>0,
$$

and the second inequality constraint is never binding in equilibrium.

Since individuals with incomes in the left tail of the distribution are constrained in the market, the resulting excess in demand will lead to an equilibrium price higher than the unconstrained solution. That is:

$$
p>\frac{q(1-\tau)}{(1-q \tau)}
$$

that in turn implies that

$$
x<1
$$

If

$$
y \geq s
$$


it follows that

$$
\left.D U\right|_{(y \geq s)} \equiv U(\text { status quo })-U(\text { redistribution }) \geq 0
$$

if and only if

$$
\tau(y-\bar{y})-(1-\tau+p \tau) b^{*}(y) \geq 0
$$

Note that $x<1$ implies that

$$
\tau-(1-\tau+p \tau) \frac{d b^{*}(y)}{d y}>0
$$

and the RHS of (2) is increasing in $y$. Since

$$
\begin{gathered}
\tau(y-\bar{y})-(1-\tau+p \tau) b^{*}(y)> \\
>\tau(s-\bar{y})-(1-\tau+p \tau) b^{*}(s)=\frac{\alpha \bar{y} \tau(1-x)+x \beta(1-\theta)(1-x)}{x \alpha}>0
\end{gathered}
$$

all agents with income $y \geq s$ will strictly prefer the status quo policy.

If

$$
y<s
$$

it follows that

$$
\left.D U\right|_{(y<s)} \geq 0
$$

if and only if

$$
\frac{1}{1-p} y-\tau \bar{y} \geq 0
$$

Since

$$
s>(1-p) \tau \bar{y}
$$

this implies that

$$
D U>0 \text { if and only if } y>(1-p) \tau \bar{y} \text {. }
$$

Therefore, the ex-post probability $\tilde{q}=F((1-p) \tau \bar{y})$ of adopting redistribution is always smaller than the ex-ante probability $q=F(\bar{y})$.

Note that the above exercise implicitly assume that agents take $q$ as given. If agents can perfectly anticipate the effect of the financial market, they will consider the expected $\tilde{q}$ in the maximization problem. Nonetheless, it can be shown that a unique equilibrium exists in which the proportion of agents in favor of redistribution is always strictly smaller 
than in the case where agents do not react to electoral uncertainty. Indeed, by applying the Implicit Function Theorem to the equation $Q(p)=0$, I can show that

$$
\left.\frac{d p}{d q}\right|_{Q(p)=0}=-\frac{\left.\frac{d Q}{d q}\right|_{Q(p)=0}}{\left.\frac{d Q}{d p}\right|_{Q(p)=0}}=\frac{\frac{d x}{d q} \int_{s} \frac{\phi(y, p)}{p x+(1-p)(1-\tau)} d F(y)}{-\left.\frac{d Q}{d p}\right|_{Q(p)=0}}>0
$$

since

$$
\frac{d x}{d q}=\frac{(1-p)(1-\tau)}{(1-\theta)(1-q)^{2} p} x^{\theta}>0 .
$$

This implies that the bounded function $F((1-p(q)) \tau \bar{y}) \in[0, F(\tau \bar{y})]$ is monotonically decreasing in $q$. Therefore the mapping

$$
q=F((1-p(q)) \tau \bar{y})
$$

has a unique fixed point. Since $(1-p) \tau \bar{y}<\bar{y}$, the result follows.

\section{Proof of Proposition 3}

Let $z_{m}$ be the expected income after elections with market and $F_{z_{m}}$ be the expected income distribution.

$$
\begin{gathered}
z_{m}=\left\{\begin{array}{rr}
(y(\tilde{y}(1-\tau)+\tau \bar{y}(1-q))+\tilde{y} q \tau \bar{y}) \frac{q \tau \bar{y}+\tilde{y}(1-\tau q)}{(\tau \bar{y}+\tilde{y}(1-\tau)) \tilde{y}} & \text { for } y \geq \gamma \\
q \tau \bar{y}+(1-q \tau) y & \text { otherwise, }
\end{array}\right. \\
F_{z_{m}}= \begin{cases}F\left(\frac{z_{m} \frac{(\tau \bar{y}+\tilde{y}(1-\tau)) \tilde{y}}{\tilde{y} \bar{y}+\tilde{y}(1-\tau q)}-\tilde{y} q \tau \bar{y}}{y(1-\tau)+\tau \bar{y}(1-q)}\right) & z_{m} \geq \bar{z} \\
F(\gamma) & z_{m} \in[q \tau \bar{y}+(1-q \tau) \gamma, \bar{z}) \\
F\left(\frac{z_{m}-q \tau \bar{y}}{1-q \tau}\right) & z_{m} \in[q \tau \bar{y}+(1-q \tau) \underline{y}, q \tau a+(1-q \tau) \gamma) \\
0 & z_{m}<q \tau \bar{y}+(1-q \tau) \underline{y},\end{cases}
\end{gathered}
$$

where $\bar{z}=\frac{(\gamma(\tilde{y}(1-\tau)+\tau \bar{y}(1-q))+\tilde{y} q \tau \bar{y})(\tau \bar{y} q+\tilde{y}(1-\tau q))}{(\tau \bar{y}+\tilde{y}(1-\tau)) \tilde{y}}$. Notice that for $y<\gamma, F_{z}$ and $F_{z_{m}}$ are identical. For $y \geq \gamma$ they cross ones in $y=\tilde{y}$ and, $F_{z}(\bar{z})>F_{z_{m}}(\bar{z})=F(\gamma)$. Since the existence of the market does not affect the mean of the income distribution:

$$
\int_{\gamma} F_{z}=\int_{\gamma} F_{z_{m}}
$$


but this implies that:

$$
\int_{\underline{y}}^{y}\left(F_{z}-F_{z_{m}}\right) d s \geq 0 \text { for all } y \in Y
$$

\section{Proof of Proposition 4}

Let $z_{m}^{\prime}$ be the expected income after elections with market, and $F_{z_{m}^{\prime}}$ be the expected income distribution when $\gamma \in(\underline{y}, \bar{y})$. In this case the end of period probability of implementing the reform $\tilde{q}$ is a function of the size the market, i.e. $\tilde{q}=F(\gamma)<F(\bar{y})=q$. Hence:

$$
\begin{gathered}
z_{m}^{\prime}=\left\{\begin{aligned}
(y(\tilde{y}(1-\tau)+\tau \bar{y}(1-\tilde{q}))+\tilde{y} \tau \bar{y} \tilde{q}) \frac{\tau \bar{y} \tilde{q}+\tilde{y}(1-\tau \tilde{q})}{(\tau \bar{y}+\tilde{y}(1-\tau)) \tilde{y}} & \text { for } y \geq \gamma \\
\tilde{q} \tau \bar{y}+(1-\tilde{q} \tau) y & \text { otherwise, }
\end{aligned}\right. \\
F_{z_{m}^{\prime}}= \begin{cases}F\left(\frac{z_{m}^{\prime} \frac{(\tau \bar{y}+\tilde{y}(1-\tau) \tilde{y}}{\tilde{y}(1-\tilde{y})+\tau \tilde{y})}-\tilde{y} \tau \bar{y}}{F(1-\tilde{y})}\right) & z_{m}^{\prime} \geq \bar{z}^{\prime} \\
F(\gamma) & z_{m}^{\prime} \in\left[\tilde{q} \tau \bar{y}+(1-\tilde{q} \tau) \gamma, \bar{z}^{\prime}\right) \\
F\left(\frac{z_{m}^{\prime}-\tilde{q} \tau \bar{y}}{1-\tilde{q} \tau}\right) & z_{m}^{\prime} \in[\tilde{q} \tau \bar{y}+(1-\tilde{q} \tau) \underline{y}, \tilde{q} \tau \bar{y}+(1-\tilde{q} \tau) \gamma) \\
0 & z_{m}^{\prime}<\tilde{q} \tau \bar{y}+(1-\tilde{q} \tau) \underline{y},\end{cases}
\end{gathered}
$$

where $\bar{z}^{\prime}=\frac{(\gamma(\tilde{y}(1-\tau)+\tau \bar{y}(1-\tilde{q}))+\tilde{y} \tilde{q} \tau \bar{y})(\tau \bar{y} \tilde{q}+\tilde{y}(1-\tau \tilde{q}))}{(\tau \bar{y}+\tilde{y}(1-\tau)) \tilde{y}}$. In this case $F_{z_{m}^{\prime}}(z)>F_{z}(z)$ for $z \leq$ $\tilde{q} \tau \bar{y}+(1-\tilde{q} \tau) \gamma$, since $\tilde{q}<q$. Moreover, $F_{z}\left(\bar{z}^{\prime}\right)>F_{z_{m}^{\prime}}\left(\bar{z}^{\prime}\right)$ if and only if

$$
G(\gamma)=\frac{(\gamma(\tilde{y}(1-\tau)+\tau \bar{y}(1-\tilde{q}))+\tilde{y} \tilde{q} \tau \bar{y})(\tau \bar{y} \tilde{q}+\tilde{y}(1-\tau \tilde{q}))}{(\tau \bar{y}+\tilde{y}(1-\tau)) \tilde{y}}-q \tau \bar{y}-\gamma(1-q \tau)>0 .
$$

But notice that

$$
\lim _{\gamma \rightarrow \underline{y}^{+}} G(\gamma)=-q \tau(\bar{y}-\underline{y})<0
$$

To get the desired result we have to check whether the two distribution functions cross only once for $z \geq \bar{z}^{\prime}$. Since

$$
\lim _{\gamma \rightarrow \underline{y}^{+}} \bar{z}^{\prime}=\underline{y}
$$

we have that for $\gamma \rightarrow \underline{y}^{+}$,

$$
F_{z_{m}^{\prime}}(z)>F_{z}(z)
$$

if and only if

$$
z<\bar{y}
$$

Therefore, by continuity there exists a $\gamma^{*}>0$ such that for $\gamma \in\left(\underline{y}, \gamma^{*}\right)$ the introduction of the electoral market increases expected income inequality for any continuous initial distribution $F$. 


\section{References}

[1] Alvarez, R.M. 1998. "Information and Elections", University of Michigan Press.

[2] Austen-Smith, David 2000. "Redistributing income under proportional representation", Journal of Political Economy 108: 1235-69.

[3] Ayers, B., C. B. Cloyd, and J. R. Robinson 2004. "Read My Lips . . .: Does the Tax Rhetoric of Presidential Candidates Affect Security Prices?" The Journal of Law and Economics, forthcoming.

[4] Bénabou, R. 2000. "Unequal Societies: Income Distribution and the Social Contract." American Economic Review 90: 96-129.

[5] Bénabou, R. and E. A. Ok 2001. "Social Mobility and the Demand for Redistribution: the POUM Hypothesis." Quarterly Journal of Economics 116: 447-487.

[6] Bertaut, C. C. and M. Starr-McCluer 2002. "Household Portfolios in the United States." In "Household Portfolios". MIT Press.

[7] Celentani, M., J. I. Conde-Ruiz, and K. Desmet 2004. "Endogenous Policy Leads to Inefficient Risk Sharing." Review of Economic Dynamics 7: 758-787.

[8] Guiso, L., M. Haliassos, and T. Jappelli 2002a. "Household Portfolios." MIT Press.

[9] Guiso, L., M. Haliassos, and T. Jappelli 2002b. "Household Stockholding in Europe: Where Do We Stand and Where Do We Go?" CSEF Working Paper 88.

[10] Herron, M. C., J. Lavin, J. Silver, and D. Cram 1999. "Measurement of Political Effects in the United States Economy: A Study of the 1992 Presidential Election." Economics \& Politics 31: 51-81.

[11] Knight, B. G. 2004. "Are Policy Platforms Capitalized Into Equity Prices? Evidence from the Bush/Gore 2000 Presidential Election." NBER Working Paper No. W10333.

[12] Lee, W. and J. E. Roemer 1999. "Inequality and Redistribution Revisited." Economic Letters 65: 339-346. 
[13] Grossman, G. M. and E. Helpman 1996. "Electoral Competition and Special Interest Politics." The Review of Economic Studies 63: 265-286.

[14] Mattozzi, A. 2004a. "Essays in Political Economy" Ph.D. Dissertation, University of Pennsylvania.

[15] Mattozzi, A. 2004b. "Can We Insure Against Political Uncertainty? Evidence from the U.S. Stock Market." Caltech Social Science Working Paper No. $120 \%$.

[16] Meltzer, A. and S. Richard 1981. "A Rational Theory of the Size of the Government." Journal of Political Economy 89: 914-927.

[17] Musto, D. K. and B. Yilmaz 2003. "Trading and Voting." Journal of Political Economy 111: 990-1003.

[18] Pantzalis, C., D. A. Stangeland, and H. J. Turtle 2000. "Political Elections and the Resolution of Uncertainty: The International Evidence." Journal of Banking and Finance 24: 1575-1603.

[19] Perotti, R. 1996. "Growth, Income Distribution, and Democracy : What the Data Say." Journal of Economic Growth 1: 149-187.

[20] Persson T., G. Tabellini, and G. Roland 2000. "Comparative Politics and Public Finance." Journal of Political Economy 108: 1121-1161.

[21] Piketty, T. 1995. "Social Mobility and Redistributive Politics." Quarterly Journal of Economics 110: 551-584.

[22] Santa-Clara, P. and R. Valkanov 2003. "The Presidential Puzzle: Political Cycles and the Stock Market." Journal of Finance, 58: 1841-1872. 
Figure 1: Demand for Electoral Securities

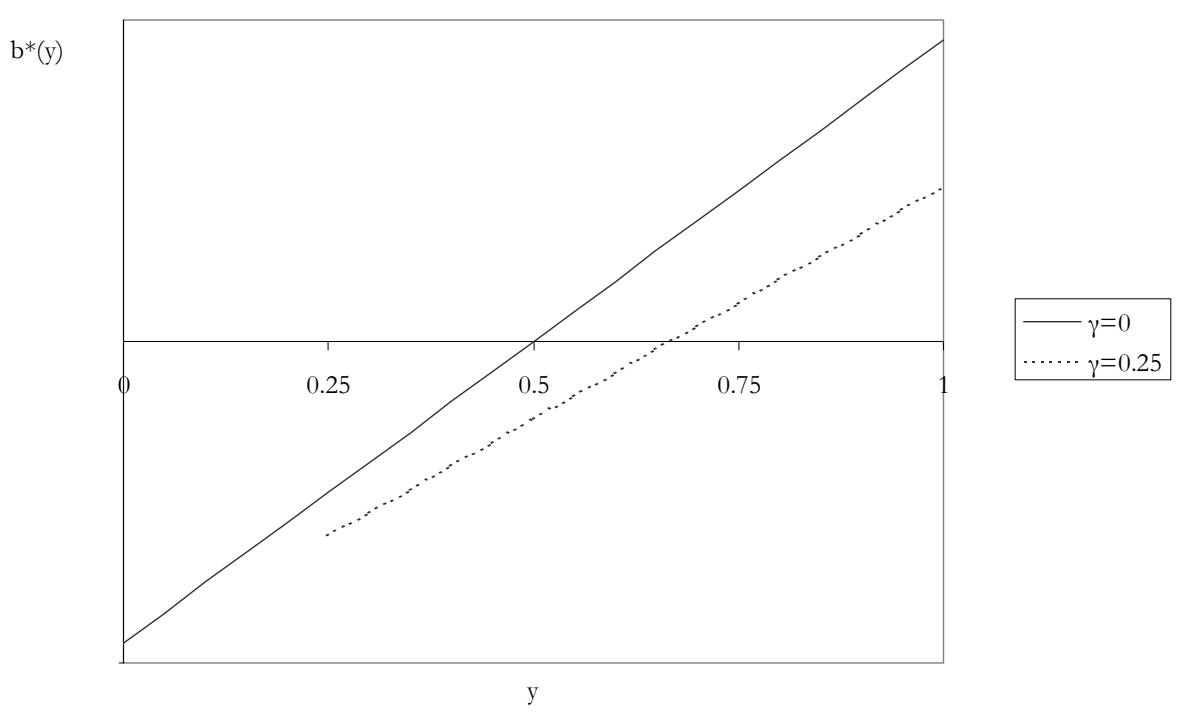

Figure 2: Preference for Redistribution

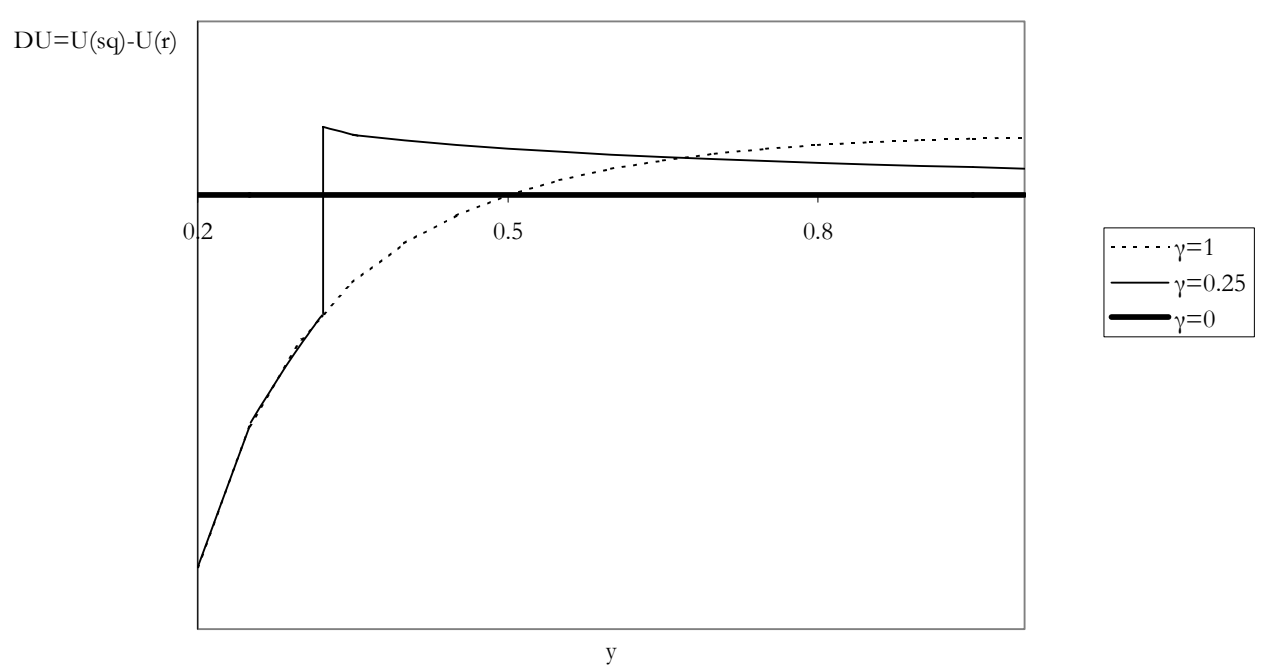


Figure 3: Expected Income Distribution

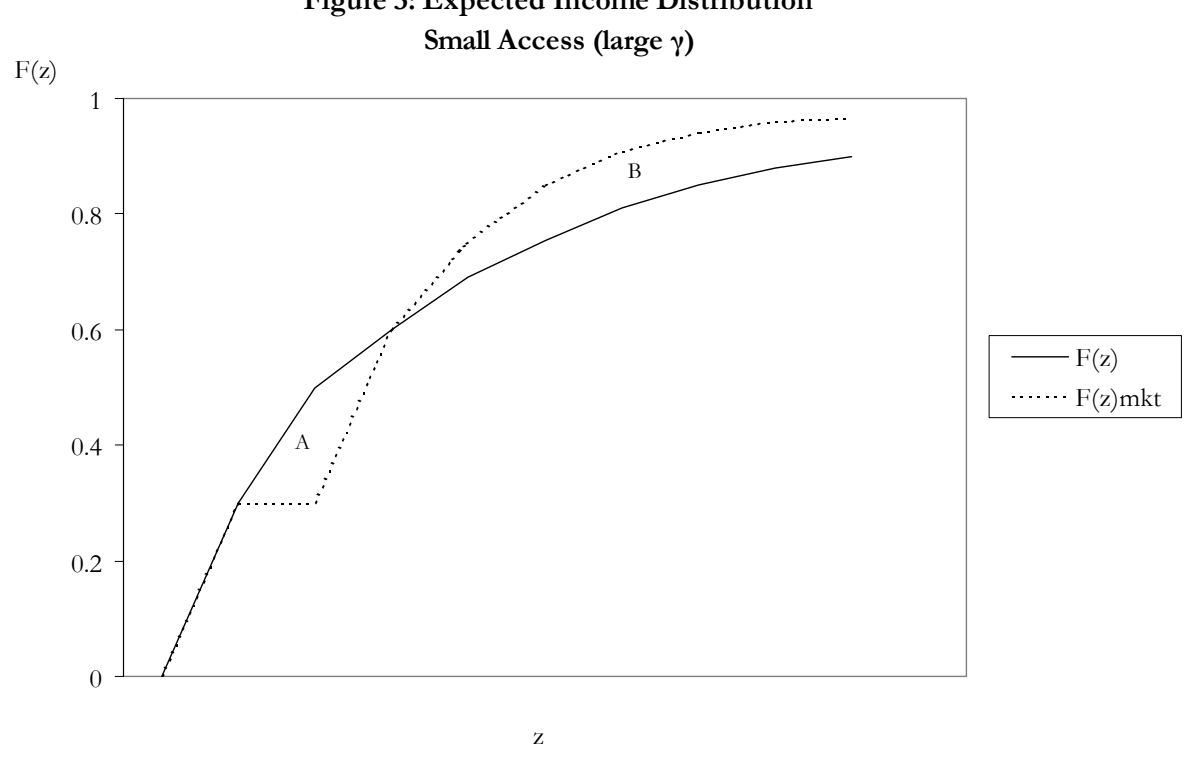


Figure 4: Expected Income Distribution Large Access (small $\gamma$ )

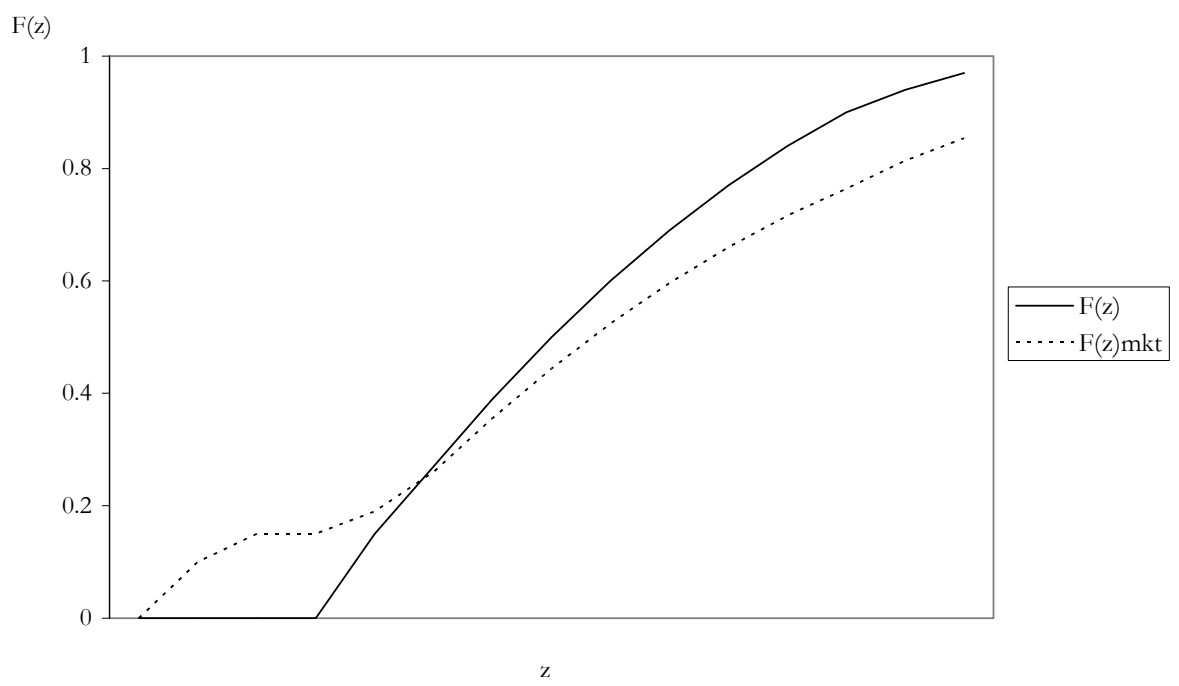

Figure 5: Redistribution and Inequality

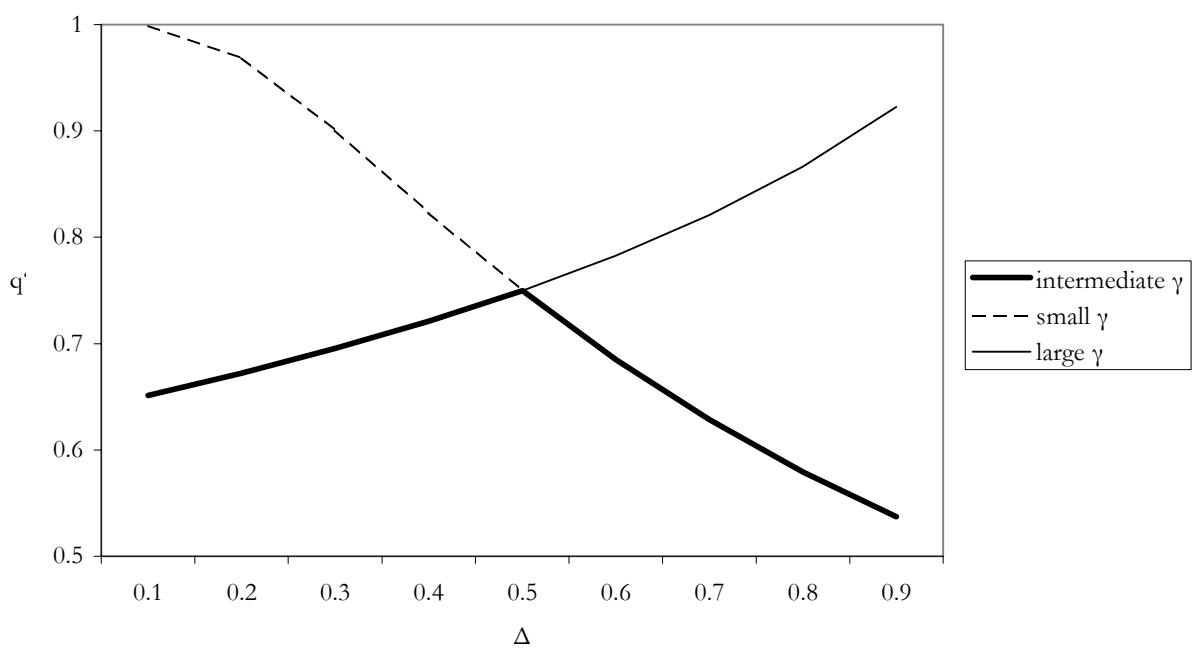


Figure 6: Inequality and Redistribution (OECD)

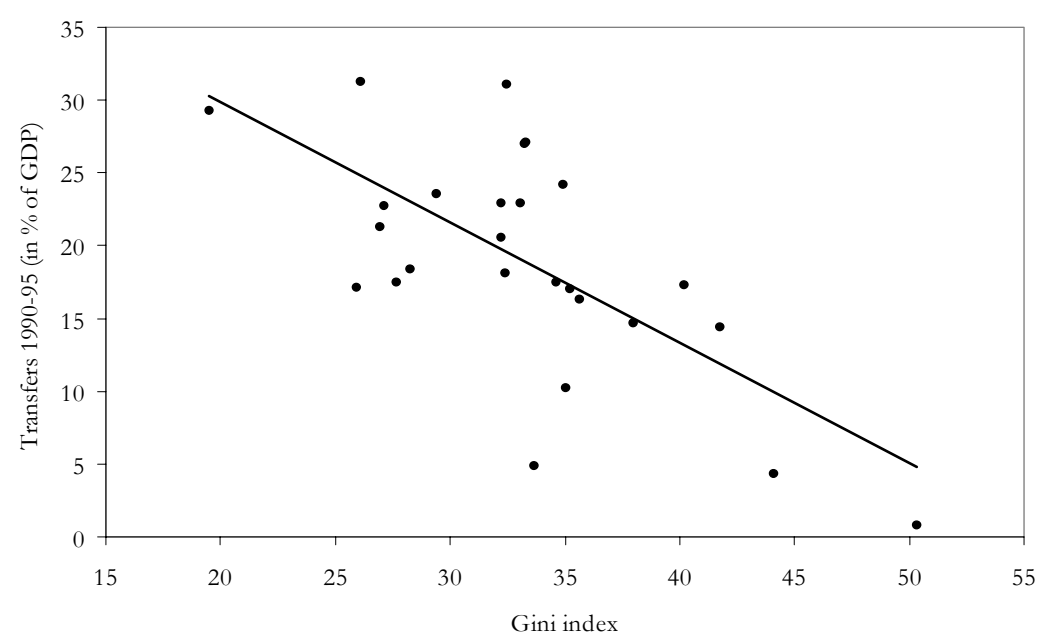

Figure 7: Stock Market and Redistribution (OECD)

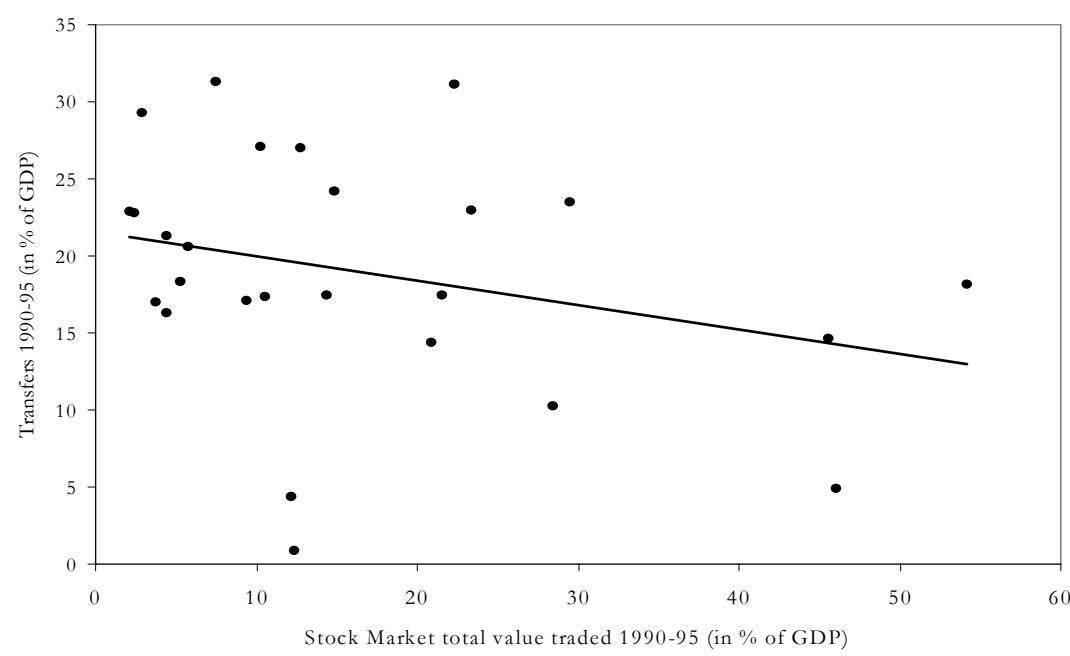

